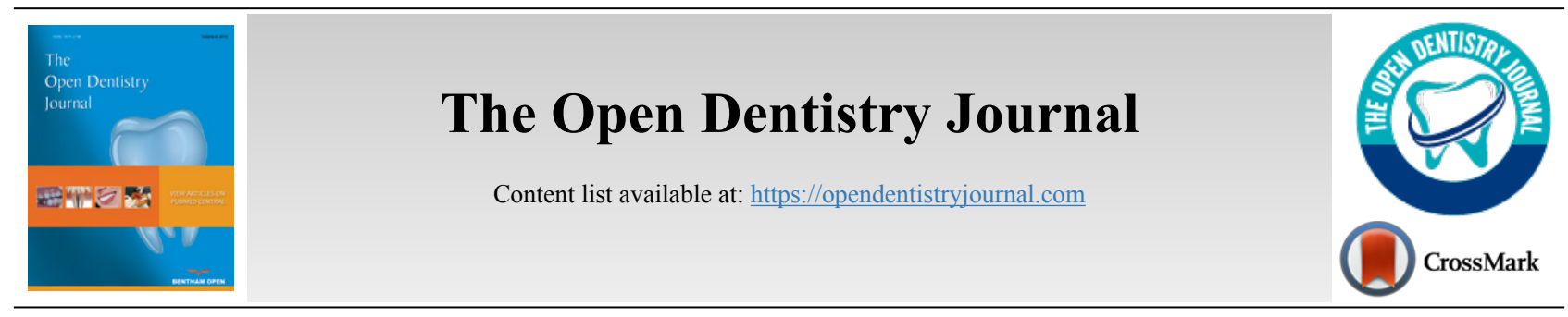

$E^{\prime}, 725, \$ /$

\title{
Coronavirus Disease 2019 (COVID-19) Pandemic Burst and Its Relevant Consequences in Dental Practice
}

\author{
Stefania Cantore ${ }^{1,2, *}$ and Andrea Ballini $i^{2,3,4}$ \\ ${ }^{I}$ Department of Interdisciplinary, Medicine University of Bari Aldo Moro, Bari, Italy \\ ${ }^{2}$ Department of Basic Medical Sciences, Neurosciences and Sense Organs, University of Bari Aldo Moro, Bari, Italy \\ ${ }^{3}$ Department of Biosciences, Biotechnologies and Biopharmaceutics, Campus Universitario “Ernesto Quagliariello”, University of Bari Aldo Moro, \\ Bari, Italy \\ ${ }^{4}$ Department of Precision Medicine, University of Campania “Luigi Vanvitelli”, Naples, Italy
}

The recent notorious burst of new coronavirus outbreak Coronavirus Disease 2019 (COVID-19) from Wuhan City, China mainland suddenly rose between November and December 2019 and has currently become a public health emergency globally as stated by the World Health Organization (WHO) on 11 March 2020 [1].

The clinical symptoms from this new viral pneumonia immediately appeared to be highly ubiquitous with fever, dry cough, headaches, severe muscular fatigue presenting abnormal chest CT, accompanied by heavy sputum secretion, hemoptysis, and diarrhea [2]. Though epidemiologically, older populations are more likely to be affected and males more than females, showing a fast worsening of general health and due to the rapidity and the aggressiveness of virus towards the respiratory system, currently, numerous youngsters have reportedly been hospitalized for COVID-19 [2]. Some of the clinical symptoms showed Covid-19 own peculiarity and distinctive traits different from related coronavirus family-like Severe Acute Respiratory Syndrome (SARS). These specific features allowed Chinese researchers to get a prompt identification of the new strain isolated directly from patients by sequencing its genome that is composed of 29,903 nucleotides [3, 4]. Everyone knows this coronavirus under the name of COVID-19, but very recently, the International Committee on Taxonomy of Viruses (ICTV) suggested the name "SARS-CoV-2" because of its phylogenetic and taxonomic structure.

Further analysis of conjunctival samples from infected cases of COVID-19 suggested that the transmission may also take place through soft mucosa and cornea. Eye exposure may provide an easy way for the virus to enter the body with small

* Address correspondence to this author at the Department of Interdisciplinary Medicine University of Bari Aldo Moro, Bari, Italy

E-mail: stefaniacantore@ pec.omceo.bari.it droplets. Notably, a few reports from Europe indicate that transmission may eventually occur through contact with asymptomatic patients [5]. Cellular infection of COVID-19 happens in a similar modality as SARS coronavirus, COVID-19 virus enters and affects through the angiotensinconverting enzyme 2 (ACE2) cell receptor and it can effectively use ACE2 as a receptor to promiscuously invade other cells, promoting the human-to-human fast transmission [6]. Outcomes from microbio-pathology assessments confirmed that the $\mathrm{ACE} 2^{+}$cells are highly present throughout the respiratory system and the cells were seen morphologically compatible with the salivary gland duct epithelium of the human oral cavity.

Therefore, the pathogenic transmission route in dental settings may take place due to the normal set-up of any dental cabinet or clinic. The inhalation of airborne micro-organisms that can remain active in the air for long periods, the procedures which require a direct approach to oral cavity of the patient and the contact with blood and different contaminated residuals generate a very easy way for virus contagion [7].

In dental practice, the transmission may thus happen through the infected droplets and aerosols from infected patients that easily remain on the whole surface area of the clinic. It was demonstrated that COVID-19 is able to survive and stay active at room temperature keeping its infectious capacity for as long as 9 days, and surviving in a well $50 \%$ humid ambiance. Preventive measures strictly suggest keeping clean and dry the whole cabinet area to contain and decrease the persistence of COVID-19 virulence.

In addition, dental healthcare givers should follow a firm protocol of procedure to immediately understand an individual's situation. Furthermore, a brief telephonic triage should be useful prior to patient's acceptance in dental care. 
According to the suggestions and precautions, the body temperature should be measured in the first place, with a contact-free forehead thermometer for the screening. A series of questions should be made before allowing the patient to the dental chair-side or during the telephonic triage. The following questions should be posed to each patient [7]:

(1) Any fever within the past 14 days? (2) Have you recently had a cough or any episode of difficulty in breathing within the past 14 days? (3) Have you traveled or have met anyone who traveled, within the past 14 days, to red zone countries or cities affected by COVID-19? (4) Have you come into contact with any individual with confirmed COVID-19 infection within the past 14 days? (5) Have you had any contact with anyone documented with fever or respiratory problems within the last 14 days? (6) Have you recently been a part of meetings, workshops or got close contact with many people?

This pandemic outbreak highlights the ongoing ability of viral spill-over from animals to cause severe disease in humans. That means robust surveillance to find, isolate, test and treat every case, to break the chains of transmission routes though the population as well as in dental clinics and hospitals. All countries have lessons to share: "We are all Italians".

\section{REFERENCES}

[1] Coronavirus disease (COVID-19) Pandemic Available from: https:// www.who.int/emergencies/diseases/novel-coronavirus-2019

[2] Zhu N, Zhang D, Wang W, et al. China novel coronavirus investigating and research team. A novel coronavirus from patients with pneumonia in China, 2019. N Engl J Med 2020; 382(8): 727-33. [http://dx.doi.org/10.1056/NEJMoa2001017] [PMID: 31978945]

[3] Chan JF, Yuan S, Kok KH, et al. A familial cluster of pneumonia associated with the 2019 novel coronavirus indicating person-toperson transmission: a study of a family cluster. Lancet 2020; 395(10223): 514-23.

[http://dx.doi.org/10.1016/S0140-6736(20)30154-9]

[PMID: 31986261]

[4] Wu F, Zhao S, Yu B, et al. A new coronavirus associated with human respiratory disease in China. Nature 2020; 579(7798): 265-9. [http://dx.doi.org/10.1038/s41586-020-2008-3] [PMID: 32015508]

[5] Rothe C, Schunk M, Sothmann P, et al. Transmission of 2019-nCoV infection from an asymptomatic contact in Germany. N Engl J Med 2020; 382(10): 970-1.

[http://dx.doi.org/10.1056/NEJMc2001468] [PMID: 32003551]

[6] Tang A, Tong ZD, Wang HL, et al. Detection of novel coronavirus by RT-PCR in stool specimen from asymptomatic child, China. Emerg Infect Dis 2020; 26(6): 1-3

[http://dx.doi.org/10.3201/eid2606.200301] [PMID: 32150527]

[7] Peng X, Xu X, Li Y, Cheng L, Zhou X, Ren B. Transmission routes of 2019-nCoV and controls in dental practice. Int J Oral Sci 2020; 12(1):

[http://dx.doi.org/10.1038/s41368-020-0075-9] [PMID: 32127517]

\section{(C) 2020 Cantore and Ballini.}

This is an open access article distributed under the terms of the Creative Commons Attribution 4.0 International Public License (CC-BY 4.0), a copy of which is available at: https://creativecommons.org/licenses/by/4.0/legalcode. This license permits unrestricted use, distribution, and reproduction in any medium, provided the original author and source are credited. 